\title{
Knowledge of Cervical Cancer and Prevalence of the Screening Services among HIV-Infected Women in Blantyre, Malawi
}

\author{
Jessie Mkandawire ${ }^{1 *}$, Flemmings Fishani Ngwira ${ }^{2}$, Fresier Chidyaonga Maseko ${ }^{1}$ \\ ${ }^{1}$ School of Public Health and Family Medicine, College of Medicine, University of Malawi, Zomba, Malawi \\ ${ }^{2}$ The Polytechnic, Language and Communication Department, University of Malawi, Zomba, Malawi \\ Email: `jessiemknda@gmail.com
}

How to cite this paper: Mkandawire, J., Ngwira, F.F. and Maseko, F.C. (2020) Knowledge of Cervical Cancer and Prevalence of the Screening Services among HIV-Infected Women in Blantyre, Malawi. Health, 12, 1496-1510.

https://doi.org/10.4236/health.2020.1211109

Received: July 10, 2020

Accepted: November 24, 2020

Published: November 27, 2020

Copyright $\odot 2020$ by author(s) and Scientific Research Publishing Inc. This work is licensed under the Creative Commons Attribution International License (CC BY 4.0).

http://creativecommons.org/licenses/by/4.0/

\begin{abstract}
HIV-infected women are at high risk of developing cervical cancer and are encouraged to undergo cervical cancer screening regularly. However, very little has been documented about the knowledge of cervical cancer and the utilization of the screening services by these women. This study aimed to investigate the knowledge of cervical cancer, prevalence of cervical cancer screening uptake and predictive factors that affect utilization of cervical cancer screening services among HIV-infected women. The study employed quantitative methods for data collection and analysis. It was conducted at Queen Elizabeth Central Hospital (QECH) from December 2017 to February 2018 where HIV-positive patients accessing QECH Antiretroviral Therapy (ART) clinic were recruited. Systematic random sampling was employed to select HIV-positive women for the survey. Data were collected using semi-structured questionnaires. STATA version 12 was used to analyze the data. Out of the 196 recruited women, the majority $(98 \% ; n=192)$ indicated having heard of cervical cancer and of the 192 women, only 57\% $(n=109)$ were able to know at least a single sign/symptom of cervical cancer, furthermore, only $47 \%$ ( $n=$ 91) indicated ever been screened, finally, getting information from health facilities and knowing at least a single sign/symptom of cervical cancer influenced HIV-infected women to go for cervical cancer screening services. The study revealed that knowledge of cervical cancer and the utilization of its screening services among HIV-infected women is low. Therefore, there is need to increase knowledge and strengthen cervical cancer screening services among HIV-infected women in the country.
\end{abstract}

\section{Keywords}

Cervical Cancer, Screening Services, HIV-Infected Women, Knowledge of 


\section{Introduction}

Cervical cancer is the second most common cancer in women worldwide. Every year more than 270,000 women die from cervical cancer globally and more than $85 \%$ of these deaths are in low and middle income countries because of poor access to screening and treatment services [1]. In Malawi, cervical cancer has become a public health problem and its trend is increasing at its frightening rate. According to World Cancer Research Fund, among the 20 African countries with high incidence of cervical cancer, Malawi had the highest cervical cancer age standardized incidence rate of 75.9/100,000 women in 2012 [2]. The burden of cervical cancer in Malawi is severe with the estimates of about three thousand, six hundred and eighty-four (3684) cases diagnosed annually of which two thousand, three hundred and fourteen (2314) women die from this disease annually [3]. The Malawi cancer statistics also indicate that cervical cancer accounted for $32.3 \%$ of all female cancers in Blantyre urban and rural population, and $22.8 \%$ of all female cancers nationally in 2000-2002 [3]. In 2007-2010 cervical cancer was the commonest cancer among women which accounted for $45.5 \%$ of the cancer cases [4]. Worst still, at least $80 \%$ of the cervical cancer cases are present in inoperable stages II-IV, and nearly all of them die [3].

Every sexually active woman aged 15 years and older has the risk of developing cervical cancer. However, women living with human immunodeficiency virus (HIV) infection have a much higher risk of human papillomavirus infection and cervical cancer than do HIV-uninfected women, because HIV-infected women are more likely to have persistent HPV infection due to immune suppression by the virus [5]. Persistent infection with HPV is necessary for the progression of cervical intraepithelial neoplasia (CIN) to invasive cervical cancer. Studies worldwide have shown a higher prevalence of CIN among HIV-infected women than HIV-negative women. For instance, a study conducted in North America revealed that HIV-infected women had a higher cervical cancer incidence rate of 26 per 100,000 person-years than HIV-negative women who had an incident rate of 6 per 100,000 person-years [6].

In a study conducted in Malawi at a national teaching hospital: Kamuzu Central Hospital $(\mathrm{KCH})$, high grade dysplasia and cervical cancer were significantly associated with HIV-infection and increasing age [7]. Results from this pathology database study at a teaching hospital showed that $24 \%$ of the HIV positive women had CIN compared to $6 \%$ of the HIV-Negative women [7]. According to the Malawi National Cervical Cancer Control Programme (MNCCCP) 2015 annual report, visual inspections with acetic acid (VIA) positive test results and cancer suspects were more common in HIV-infected women than in HIV negative women; $15 \%$ compared to $7.9 \%$ respectively [8]. Almost similar results were 
also obtained in the MNCCCP 2013 annual report [9]. This indicates that there is a great need for HIV-infected women to utilize the cervical cancer screening services for early diagnosis and treatment of cervical cancer.

World Health Organization recommends women known to be HIV-infected or living in high HIV prevalence areas to go for cervical cancer screening despite their age, as long as these women are sexually active [1]. Malawi is one of the countries, in Sub-Saharan Africa with high HIV prevalence estimated at 10.6\% [10]. The prevalence is high among females (12.9\%) compared to males $(8.1 \%)$. However, despite the campaigns and opening up of several cancer screening services by the country's ministry of health at various health facilities, access and utilization of cancer screening services among women in the general population remain a problem and the situation is worse among HIV-positive women [8]. It is alarming that women at high risk of developing cervical cancer fail to utilize the screening services. Several studies have reported underutilization of cervical cancer among HIV-infected women and even the general population of women in many countries. For instance, a study conducted in Uganda of which 900 women participated, only 43 (4.8\%) reported to have ever been screened for cervical cancer [11]. A study done by Matangaidze, Olivia, Ramalivhana, Augustine and Maxwell [12] in Zimbabwe reported that although 77.4\% of HIV-infected women felt being at risk of developing cervical cancer, only $9 \%$ had ever had a screening test. Similarly, a study done by Ezechi, Gab-okafor, Ostergren and Pettersson [13] in Nigeria, prevalence of cervical cancer screening among HIV-infected women was reported to be as low as $9.4 \%$.

Access and utilization of cervical cancer services by all women are vital in the control and prevention of cervical cancer. However, several individual and health system-related factors have been reported as barriers to cervical cancer screening by the general population of women. Literature reveals that limited knowledge on their susceptibility to cancer, its symptoms and signs, are the major factors influencing people's decisions [4] [14] [15]. Research on cervical cancer prevention in Malawi further indicates that factors affecting low turn-ups in seeking preventative cancer screening include low perceived susceptibility, misconceptions about the screening process, cultural beliefs, lack of symptoms and low perceived benefits from the services [14] [15] [16] [17] [18]. A qualitative study conducted in Chiradzulu-Malawi [19] reported similar factors of lack of knowledge to cervical cancer and its risk factors as barriers to access and utilization of cervical cancer. In a study conducted in Mulanje, low knowledge levels, low perceived benefits, low perceived susceptibility, cultural beliefs and misconception of the disease were discovered to be the barriers to seeking cervical cancer screening services among women [17]. Thinking inductively from the reasons for patients' delay and low turn-ups in seeking medical care, one may infer that people lack proper knowledge to enhance their decision making.

The Health Belief Model (HBM) postulates that individuals will act to protect or promote their health if they believe they are susceptible to the disease, the consequences of the disease are severe and the benefits of preventing outweigh 
the cost or barriers [20]. Using the health belief model to guide the current study, the utilization of cancer screening services is an act that women do to protect and promote their health as far as cervical cancer is concerned, and they act in such a way, if they are fully aware of the severe consequences of the disease and the benefits of taking such an action. Therefore, awareness, which brings about the knowledge is very important in cervical cancer care. Despite HIV-infected women being at high risk of developing cervical cancer, there is little information regarding specific issues that affect them. Studies that have been conducted on barriers to utilization of cervical cancer screening services only targeted the general population of women in Malawi [13] [14] [15] [17] [18] [19]. Therefore, this study aimed to provide specific evidence on the knowledge of cervical cancer in terms of signs and risk factors, prevalence of cervical cancer screening uptake and predictive factors that affect utilization of cervical cancer screening services among HIV-infected women. Specifically, the study was aimed to provide answers to the following research questions:

1) What is the level of knowledge of cervical cancer among HIV-infected women?

2) What is the prevalence of cervical cancer screening uptake by HIV-infected women?

3) What are the predictive factors that influence the utilization of cervical cancer screening services among HIV-infected women?

\section{Methods}

\subsection{Study Design and Population}

The current study was a facility based descriptive cross-sectional survey that used quantitative data collection and analysis methods. The study was conducted at Queen Elizabeth Central hospital (QECH), which is one of the referral and teaching hospitals in Malawi, hence it provided a better environment for the study; we got participants from different areas inside and outside Blantyre city. The study population comprised of HIV-infected women who at the time of study were accessing QECH ART clinic. All HIV-infected women, 18 years and above, whom at the time of study were accessing ART clinic at QECH, were eligible for the study. All critically ill women were excluded from participating. The survey was conducted within a period of three months, from December 2017 to February 2018.

\subsection{Sample Size Determination and Selection}

The sample size was calculated using the following formula: $n=\frac{z^{2} p(1-p)}{m^{2}}$; where, $n=$ sample size, $z=z$ value (e.g. 1.96 for 0.05 significance level), and $m=$ marginal error of 0.05 . The $p$ stands for proportion of HIV-infected women utilizing the cervical cancer screening, which according to MNCCCP 2015 annual report was at $15.8 \%$ [8]. Systematic random sampling was employed to select 196 
HIV-positive women for quantitative data.

\subsection{Data Collection and Management}

Data were collected using a semi-structured questionnaire which was administered by the researcher. Quality of data collection tools was achieved by pretesting and making necessary corrections before the data collection. The survey was conducted after the participants accessed the medical care and drugs. Questionnaires were checked for completeness, consistency and accuracy. The survey had six closed-ended questions and 12 open-ended items which assessed participants' knowledge of cervical cancer, perceived susceptibility, and access and utilization of cervical cancer screening services. Responses from the open-ended questions were first coded qualitatively, grouping similar ideas together, and afterwards, the patterns were assigned codes to be analysed quantitatively. The data was entered in an excel sheet and later imported into STATA version 12 for cleaning and analysis.

\subsection{Data Analysis}

Descriptive statistics were used to socio-demographic characteristics of the participants and to describe HIV-infected women utilizing cervical cancer screening services. Univariate analysis using Chi-square test was performed to identify factors associated with the utilization of the screening services. Multivariate logistic regression was used to assess the significance of the utilization of cervical cancer screening services and independent variables. Variables were entered into a model if their $p$-value on the univariate analysis were 0.05 or $<0.05$. Odds ratio and their $95 \%$ confidence intervals (CI) were used to measure strength of association. A $p$-value of $<0.05$ was considered significant. To ensure the instruments' internal consistency, reliability analysis using Cronbach's alpha was calculated and the overall internal reliability of the instrument was alpha 0.76 .

\subsection{Ethical Considerations}

College of Medicine research and ethics committee (COMREC) approved the research protocol. Further authorization was obtained from the Hospital Director of QECH. Verbal and written informed consents were sought from the participants prior to conducting the study. Interviews were conducted in a closed room for privacy and confidentiality. Anonymity was ensured through the use of identity numbers.

\section{Findings}

\subsection{Socio-Demographic Characteristics of Participants}

The study recruited a total of 196 respondents who were HIV infected women attending the ART clinic at Queen Elizabeth Central Hospital. The mean age of the study participants was 34 . On education, $40.3 \%(n=79)$ had attended primary education, $42.9 \%(n=84)$ secondary education, $7.7 \%(n=15)$ tertiary 
education and the rest, 9.2\% $(n=18)$ had no formal education. Over half of the respondents, $57.7 \%(n=113)$ were married, but $24 \%(n=47)$ were widowed, $14.8 \%(n=29)$ were divorced and only $3.6 \%(n=7)$ had never been married. The majority of participants were business women $44.9 \%(n=88)$, while $32.1 \%$ $(n=63)$ were unemployed and only $23 \%(n=45)$ were employed.

\subsection{Knowledge and Prevalence of Cervical Cancer Screening}

Out of the 196 women recruited in the study, 98\% $(n=192)$ indicated having ever heard of cervical cancer. Out of 192 women who indicated having heard of cervical cancer, $53 \%(n=101)$ reported that they got cervical cancer information from the health facility, $36 \%(n=70)$ from the media, $8 \%(n=16)$ from relatives or friends, and $3 \%(n=5)$ from both media and health facility. Furthermore, $57 \%(n=109)$ were able to know at least a single sign/symptom of cervical cancer such as bleeding after menopause, increased vaginal discharge and pain during intercourse, and $43 \%(n=83)$ were not sure or did not know any sign/symptom of cervical cancer. Out of 192 women only $47 \%(n=91)$ indicated ever been screened, while 53\% $(n=101)$ indicated never been screened for cervical cancer. Table 1 shows the number of women who indicated having heard about cervical cancer and those who indicated having ever been screened subcategorised by socio-demographic characteristics.

Participants were further subcategorised based on other factors that would influence them into cervical cancer screening. These factors were "source of cervical cancer information", "knowledge of signs/symptoms of cervical cancer", and "perceived susceptibility". Most women $(n=101)$ reported health facility to be their source of cancer information and regarding knowledge of cervical cancer, most women $(n=82)$ indicated not knowing the symptoms and signs of cervical cancer. Table 2 shows the number of women who indicated having

Table 1. Social demographic characteristics of women ever heard of cervical cancer $(n=$ 192).

\begin{tabular}{cccc}
\hline Social demographic characteristic & Ever heard & Ever screened \\
\hline Age group & $18-28$ & 16 & $06(37.5 \%)$ \\
$29-39$ & 80 & $33(41.25 \%)$ \\
& $40-50$ & 62 & $30(48.39 \%)$ \\
Education & $51+$ & 34 & $22(64.71 \%)$ \\
& None & 17 & $06(35.29 \%)$ \\
& Primary & 77 & $32(41.56 \%)$ \\
Marital status & Secondary & 83 & $45(54.22 \%)$ \\
& Tertiary & 15 & $08(53.33 \%)$ \\
& Married & 111 & $48(43.24 \%)$ \\
& Single & 7 & $04(57.14 \%)$ \\
& Widowed & 46 & $26(56.52 \%)$ \\
& Divorced & 28 & $13(46.43 \%)$ \\
\hline
\end{tabular}


Table 2. Proportion of women ever heard of cervical cancer by influencing factors $(n=$ 192).

\begin{tabular}{|c|c|c|c|}
\hline \multicolumn{2}{|c|}{ Influencing factors } & \multirow{2}{*}{$\begin{array}{c}\text { Ever heard } \\
101\end{array}$} & \multirow{2}{*}{$\begin{array}{c}\text { Ever screened } \\
56(55.45 \%)\end{array}$} \\
\hline \multirow{4}{*}{ Source of information } & Health facility only & & \\
\hline & Media only & 70 & $25(35.71 \%)$ \\
\hline & Friends/relatives & 16 & $6(37.50 \%)$ \\
\hline & Health facility and Media & 5 & $4(80.0 \%)$ \\
\hline \multirow{6}{*}{$\begin{array}{l}\text { Knowledge of Cervical } \\
\text { cancer symptoms/signs }\end{array}$} & Breeding & 46 & $28(60.87 \%)$ \\
\hline & Virginal discharge & 13 & $10(76.92 \%)$ \\
\hline & Pain during intercourse & 9 & $5(55.56 \%)$ \\
\hline & Abnormal cells around the cervix. & 18 & $11(61.11 \%)$ \\
\hline & No clear signs in early stages & 23 & $10(43.48 \%)$ \\
\hline & Don't know/Not sure & 82 & $27(32.53 \%)$ \\
\hline \multirow{3}{*}{ Perceived susceptibility } & High & 58 & $33(56.90 \%)$ \\
\hline & Medium & 93 & $40(43.01 \%)$ \\
\hline & Low & 36 & $14(38.89 \%)$ \\
\hline
\end{tabular}

heard about cervical cancer and those who got screened of it based on the influencing factors.

\subsection{Associations between Independent Factors and Cervical Cancer Screening}

To establish associations between independent variables and cervical cancer screening, Chi-square tests were used. The independent variables employed for analysis were socio-demographic variables and other influencing variables of source of information, knowledge of signs and symptoms of cervical cancer, and women's perceived level of susceptibility. The measure of association was done using a $p$-value of 0.05 . Chi-square test results showed no statistical significant association between socio-demographic factors and cervical cancer screening. However, significant relationships were found between cervical cancer screening and source of information ( $\left.X^{2}=9.22, p=0.027\right)$, and knowledge of cervical cancer signs/symptoms ( $\left.X^{2}=16.99, p=0.005\right)$. Independent variables associated with the cervical cancer screening in Chi-square analysis, were analysed further using logistic regression and the results are tabulated in Table 3.

\subsection{Interpretation of Logistic Regression Results}

The logistic regression results were interpreted in terms of the Odds Ratio. Each independent variable was interpreted based on the reference category in that group. Women who heard the information from both the health facility and media were 3 times more likely to have cervical cancer screening test than those who heard the information from the health facility only [OR $=3.21,95 \% \mathrm{CI}(0.35$ - 29.78), $p=0.304]$. On the other hand, women who heard the information from 
Table 3. Factors influencing access and utilization of cervical cancer screening services.

\begin{tabular}{ccccc}
\hline Variable & & OR & $p$-value & 95\% CI \\
\hline \multirow{3}{*}{ Source of information } & Health facility & $1(\mathrm{RC})$ & & \\
& Media & 0.45 & 0.012 & $0.24-0.84$ \\
& Relatives/Friends & 0.48 & 0.188 & $0.16-1.44$ \\
& Health facility and Media & 3.21 & 0.304 & $0.35-29.78$ \\
& Bleeding & $1 \mathrm{RC}$ & & \\
Knowledge of Cervical & Pain during intercourse & 0.80 & 0.766 & $3.40-8.86$ \\
& Abreased vaginal discharge & 2.14 & 0.293 & $0.52-8.86$ \\
& Abnormal cells on the cervix & 1.01 & 0.990 & $0.18-1.36$ \\
& No clear early signs & 0.49 & 0.174 & $0.18-1.36$ \\
& Don't know/Not sure & 0.31 & 0.002 & $0.15-0.66$ \\
\hline
\end{tabular}

$\mathrm{RC}=$ Reference category

the media only had $55 \%$ reduced odds of cervical cancer screening than those who heard the information from the health facility [OR $=0.45,95 \% \mathrm{CI}(0.24$ $0.84), p=0.012$ ]. Furthermore, those who heard the information from relatives or friends had $52 \%$ reduction in the odds of cervical cancer screening compared with those who heard the information from the health facility $[\mathrm{OR}=0.48$, 95\%CI (0.16 - 1.44), $p=0.304]$.

Women who reported knowing increased virginal discharge as a symptom of cervical cancer were 2.1 times more likely to have a cervical cancer screening test compared with women who knew bleeding. On the other hand, women who knew pain during intercourse as a symptom of cervical cancer had $20 \%$ reduction in the odds of having cervical cancer screening compared with those who knew bleeding. Furthermore, women who reported that cervical cancer has no clear sign in its early stages and those who did not know any sign/symptom of cervical cancer had 51\% [OR $=0.49,95 \% \mathrm{CI}(0.18-1.36), p=0.174]$ and $69 \%$ $[\mathrm{OR}=0.31,95 \% \mathrm{CI}(0.15-0.66), p=0.002]$ reduction in the odds of having cervical cancer screening respectively, compared with women who knew bleeding as a sign. In addition, the results showed that there was a statistically significant difference in the odds of having cervical cancer screening test among women who knew bleeding as a symptom of cervical cancer compared with those who did not know any sign/symptom ( $p=0.002)$. The results suggest that knowing signs and symptoms of cervical cancer is vital in the access of screening services. Another statistical significant difference was observed in the odds of getting information from the hospital compared with the media only $(p=0.012)$ which suggests that getting information from the health experts is paramount for the utilization of screening services.

\section{Discussion}

The present study aimed to provide specific evidence on the knowledge of 
cervical cancer in terms of warning signs and risk factors, prevalence of cervical cancer screening uptake and predictive factors that affect utilization of cervical cancer screening services among HIV-infected women. To begin with, demographic characteristics of the current sample can already provide some insights into the cervical cancer message reception and the utilization of screening services by these HIV-infected women. On education, for instance, out of 196 participants, almost 50\% $(n=97)$ had primary school or no formal education. As this was a representative sample of the population, we could conclude that almost half of the HIV-infected women in the study population fall into this category and might be unable to benefit from health promotion messages especially in print media, even when the messages were to be written in their own local language. As Peterson, Dwyer, Mulvaney, Dietrich and Rothman [21] argued, low literacy levels associate with less knowledge about cervical cancer and its screening services. This already calls for a change of communication strategy for such women to be knowledgeable with cervical cancer rather than using strategies that would require higher literacy levels to understand the material.

The current study indicates that almost all women (98\%) who participated in the study had ever heard of cervical cancer. However, a further investigation reveals that the women lacked comprehensive knowledge of cervical cancer as most of them were not sure of or did not know any sign/symptom of cervical cancer. This suggests that the information they get is not comprehensive enough for them to know the disease. Their lack of comprehensive knowledge about the disease might have also resulted from their low literacy levels, which makes them fail to comprehend health promotion messages especially for those that require them to use their literacy levels such as reading and comprehending complex cancer messages [21]. These findings are consistent with findings of other studies done among HIV-infected women and the general population of women in Malawi, Zimbabwe, Nigeria, South Africa and several African countries where comprehensive knowledge of cervical cancer was low [12] [22] [23].

Regarding sources of information, the study reveals that the majority (53\%) reported to have heard their cancer information from the health personnel. These findings indicate that health facilities are the major sources of cervical cancer information among HIV-infected women. This is consistent with other studies done in Malawi, Nigeria and Ethiopia among HIV-women and the general population of women [22] [23] [24], where they also found that health professionals are the major sources of cervical cancer information as most women indicated to have ever heard of cervical cancer from them. Compared with men, women are more likely to visit the hospital especially during their reproductive years due to their management of gynecological and reproductive issues. It is at the clinic that they have more chance of getting cancer information than elsewhere. Women often trust health information when it comes from the experts themselves like doctors and nurses or people trained by medical professionals [18]. Therefore, it is rational to note that they regard information from health professional as vital. 
On prevalence of cervical cancer screening among HIV-infected women, the study shows that only $47 \%$ of the HIV-infected women had ever had a cervical cancer screening test. This indicates that access and utilization of the screening services among these women is still low despite its advocacy on radios and wherever these women get cervical cancer information. These results are similar to the results of the studies done in other African countries like Ethiopia and Cameroon [24] [25]. The prevalence of cervical cancer screening, however, has shown to increase with the increase in the level of education one has attained, where more women who indicated to have secondary and tertiary education had ever had a cervical cancer screening test compared to women with primary school and no formal education. The possible explanation for this might be the fact that education improves understanding of health issues and promotes better health seeking behavior among individuals, as such, education is linked with patient's self-perception of cervical cancer and understanding health education and counselling that goes on with it [13].

Interestingly, a similar pattern has also been found on age where the prevalence of cervical cancer screening has shown to increase with the increasing age of the participants. The results are in line with study finding found in Kenya among the general population of women, where women in the age group 40 - 49 were 6 times more likely to have a screening test than young women (age group 18 - 25) [26]. This demonstrates the belief that those who are younger do not really feel susceptible of the disease and as a result, they feel no need for screening. Health education in Malawi should aim at ironing out this kind of wrong perception among young women.

In the current study, almost $58 \%(n=113)$ were married and literature suggests that married women are more likely to use cervical cancer screening services than single, divorced or widowed women [18]. This means that being married, or simply living with a partner, women gain advantage of being encouraged by their partners to go for cancer screening. Contrary to our expectation, married women in this study ranked the least (43\%) on the prevalence of cervical cancer screening than their counterparts: divorced (46\%), widowed $(57 \%)$ and single (57\%). The current results certainly indicate that living with a partner does not make women advantageous over fellow women without their life partners. Husbands do not encourage their wives to attend screening services, and conversely, according to the results, they might as well discourage them to go for screening. Health advocates on cervical cancer need to advise husbands, especially of HIV-infected women to encourage their spouses for cervical cancer screening. To achieve that, advocates need to also make sure husbands fully understand the disease and the consequences of not utilizing cancer screening services.

Logistic regression results show that women who heard the information from the media only were less likely to have a cervical cancer screening test compared with those who heard it from the health facility. This entails that messages of cervical cancer screening services through media should critically be looked at, 
in as far as increasing access and utilization of the cervical cancer screening services is concerned. The media should aim at helping women be equipped with information about cervical cancer which in turn would increase access and utilization of cervical cancer screening, therefore, it should broadcast comprehensive cervical cancer messages. Since interpersonal communication entails "how" the message is being said in order to establish a good interpersonal relationship with the other person; studies have found the health facility to be more effective on knowledge of cancer as compared to mass media [27] [28]. Women meet health professional who talks to them about the disease in a very interpersonal manner. It is, therefore, inevitable for women to indicate health facilities as their source of information.

Furthermore, logistic regression reveals that women who knew bleeding and other symptoms of cervical cancer were more likely to utilize cervical cancer screening services than women who did not know any sign. This suggests that knowledge of at least a sign/symptom of cervical cancer can influence one's decision of accessing the screening services. Other studies done elsewhere found similar results: knowledge of risk factors and signs of cervical cancer emerge to be an important factor in women's preventive measures such as going for screening services [29] [30]. This study therefore postulates that increasing knowledge about cervical cancer signs/symptoms should be a priority in trying to detect cervical cancer early. Knowledge deficit poses a major threat to individuals who are at risk of developing cervical cancer, as they cannot access preventive measures. The current study has discovered that very few women know the disease comprehensively. This suggests that many HIV-infected women fall into this category where lack of knowledge would lead them into being ignorant of the disease and the protective measures. As a result of inadequate knowledge, women end up suffering at home without presenting their cases to the hospital, which leads to late presentation of the disease [14]. There is need for health advocates to equip people with the right knowledge for their own health protection.

The insight that we can get from these findings is that, it is important for one to comprehensively know cervical cancer for her to take necessary steps towards her health protection behavior such as screening [29] [30]. It is this knowledge that makes one know how susceptible she is, the signs and symptoms of the disease, its risk factors, its preventive measures and its early detection services. Without knowledge, even if one becomes sick, they would not even think of it to be cervical cancer, hence no need of going for cervical cancer screening. Therefore, health education which aims at equipping people with knowledge of the signs/symptoms of cervical cancer is required. In this study some women perceived themselves as being at risk of developing cervical cancer due to their HIV-positive status, which shows they are aware that HIV is a risk factor for cervical cancer. In addition, participants in this study indicated that they were encouraged to go for cervical cancer screening because of the knowledge they acquired through health education during their visits to health facilities. This 
postulates that health workers have a major role to influence women to access and utilize cervical cancer screening services as other researchers have also suggested [30].

\subsection{Study Limitations and Further Research}

The findings of the current study are, however, subject to some limitations. First, the study was facility-based, done at only one hospital; this may affect generalization of the results to the general population of HIV-infected women. Future research should consider a wide coverage of the sample to at least cover a reasonable area within the country. Second, the study did not triangulate the information given by HIV-infected women with health workers providing the screening services since the screening services are provided outside the study setting (ART clinic). Further studies would do well if they would include health workers in their methodologies.

\subsection{Implications for Cervical Cancer Advocates}

Limitations aside, this study found that although most women ever heard of cervical cancer, comprehensive knowledge of cervical cancer is low. This in turn affected their access and utilization of the screening services. According to HBM, individuals will act to protect or promote their health if they believe they are susceptible to the disease [20]. It should be noted, therefore, that for HIV-infected women to know cervical cancer; they need to understand the signs and symptoms of the disease and its risk factors, know how susceptible they are, see the benefits of going for screening and understand the consequences of not utilizing the screening services. As a result, they will have efforts to prevent the disease by avoiding exposure to risk factors and utilizing screening services to protect their health. Therefore, in order to improve access and utilization of cervical cancer screening among HIV-infected women, strategies such as integrating cervical cancer into the ART clinic and increasing coverage of cervical cancer messages and education in communities would help.

These findings suggest that there is a great need to increase knowledge of cervical cancer among HIV-infected women. Health education about cervical cancer should be a continuous process because it is through education that people can be equipped with comprehensive knowledge about cervical cancer which in turn may improve access and utilization of the screening services. This health education should not only be provided in health facilities but also in the communities in which these people leave. This can be achieved by making use of the women within the communities as peer educators. Furthermore, integrating cervical cancer screening services into the ART clinic would be a motivation for many HIV-infected women. The results indicate that women trust the health information from health personnel. Therefore, if the information of cervical cancer can go together with the screening in the ART, more women would go for cervical cancer screening as they would want to put into practice the infor- 
mation provided to them right there in the ART Clinic.

\section{Conflicts of Interest}

The authors declare no conflicts of interest regarding the publication of this paper.

\section{References}

[1] World Health Organization (2013) March 2013 Edition Comprehensive Cervical Cancer Revention and Control. World Health Organization, Geneva. http://apps.who.int/iris/bitstream/10665/78128/3/9789241505147

[2] World Cancer Research Fund International (2014) Cervical Cancer Statistics. World Cancer Research Fund International, London.

http://www.wcrf.org/int/cancer-facts-figures/data-specific-cancers/cervical-cancer-s tatistics

[3] Government of Malawi (2005) National Service Delivery Guidelines for Cervical Cancer Prevention. Ministry of Health, Lilongwe.

[4] Msyamboza, K.P., Dzamalala, C., Mdokwe, C., Kamiza S. and Lemerani, M. (2012) Burden of Cancer in Malawi, Common Types, Incidence and Trends: National Population-Based Cancer Registry. BMC Research Notes, 5, Article No. 149. https://doi.org/10.1186/1756-0500-5-149

[5] Odendal, L. (2011) Cervical Cancer in Women with HIV. HIV and AIDS in Practice, 174, 314-320.

[6] Alison, G.A. (2013) Invansive Cervical Cancer among HIV-Infected Women. Journal of Acquired Immune Deficiency Syndrome, 62, 405-413. https://doi.org/10.1097/qai.0b013e31828177d7

[7] Kohler, R.E., Tang, J., Gopal, S., Chinula, L., Hosseinipour M.C. and Liomba, G.N. (2016) High Rates of Cervical Cancer among HIV-Infected Women at a Referral Hospital in Malawi. International Journal of STD AIDS, 27, 753-760. https://doi.org/10.1177/0956462415592999

[8] Malawi Ministry of Health (2016) Malawi National Cervical Cancer Control Programme Annual Review 2015. Ministry of Health, Lilongwe.

[9] Malawi Ministry of Health (2014) Malawi National Cervical Cancer Control Programme Annual Review 2013. Ministry of Health, Lilongwe.

[10] National AIDS Commission (2015) National HIV and AIDS Research Strategy 2014-2016. National AIDS Commision, Lilongwe.

[11] Ndejjo, R., Mukama, T., Musabyimana, A. and Musoke, D. (2016) Uptake of Cervical Cancer Screening and Associated Factors among Women in Rural Uganda: A Cross Sectional Study. PLoS ONE, 11, e0149696.

https://doi.org/10.1371/journal.pone.0149696

[12] Olivia, M., Ramalivhana, N.J., Augustine, N.P.N. and Maxwell, M. (2016) Knowledge, Attitudes and Practices of HIV-Infected Women on Cervical Cancer Screening at a Church-affiliated Hospital. IOSR Journal of Dental and Medical Sciences, $15,119-126$.

[13] Ezechi, O.C., Gab-okafor, C.V., Ostergren, P.O. and Pettersson, K.O. (2013) Willingness and Acceptability of Cervical Cancer Screening among HIV Positive Nigerian Women. BMC Public Health, 13, Article No. 46. https://doi.org/10.1186/1471-2458-13-46

[14] Chadza, E., Chirwa, E., Maluwa, A., Malata, A., Kazembe, A. and Chimwaza, A. 
(2012) Factors That Contribute to Delay in Seeking Cervical Cancer Diagnosis and Treatment among Women in Malawi. Health, 4, 1015-1022. https://doi.org/10.4236/health.2012.411155

[15] Msyamboza, K.P., Phiri, T., Sichali, W., Kwenda, W. and Kachale, F. (2016) Cervical Cancer Screening Uptake and Challenges in Malawi from 2011 to 2015: Retrospective Cohort Study. BMC Public Health, 16, Article No. 806.

https://doi.org/10.1186/s12889-016-3530-y

[16] Maseko, F.C., Chirwa, M.L. and Muula, A.S. (2015) Underutilization of Cervical Cancer Prevention Services in Low and Middle Income Countries: A Review of Contributing Factors. Pan African Medical Journal, 21, Article 231. https://doi.org/10.11604/pamj.2015.21.231.6350

[17] Fort, V.K., Makin, M.S., Siegler, A.J., Ault, K. and Rochat, R. (2011) Barriers to Cervical Cancer Screening in Mulanje, Malawi: A Qualitative Study. Patient Preference and Adherence, 5, 125-131. https://doi.org/10.2147/PPA.S17317

[18] Hami, M.Y. and Ehlers, V.J. (2014) Women's Perceived Susceptibility to and Utilisation of Cervical Cancer Screening Services in Malawi. Health SA Gesondheid, 19, 1-8. https://doi.org/10.4102/hsag.v19i1.787

[19] Ports, K.A., Reddy, D.M. and Rameshbabu, A. (2014) Cervical Cancer Prevention in Malawi: A Qualitative Study of Women's Perspectives Cervical Cancer Prevention in Malawi: A Qualitative Study of Women's Perspectives. Journal of Health Communication, 20, 94-104. https://doi.org/10.1080/10810730.2014.908986

[20] Glanz, A., Glanz, K., Rimer, B.K. and Lewis, F.M. (2002) Health Behaviour and Health Education: Theory Research and Practice. 3rd Edition, Jossey-Bass, San Francisco.

[21] Peterson, N.B., Dwyer, K.A., Mulvaney, S.A., Dietrich, M.S. and Rothman, R.L. (2007) The Influence of Health Literacy on Colorectal Cancer Screening Knowledge, Beliefs and Behavior. Journal of the National Medical Association, 99, 1105-1112.

[22] Abiodun, O.A., Fatungase, O.K. and Awosile, J.O. (2013) An Asessment of Women's Awareness and Knowledge about Cervical Cancer and Screening and the Barriers to Cervical Screening in Ogun State, Nigeria. IOSR Journal of Dental and Medical Science, 10, 52-58. https://doi.org/10.9790/0853-1035258

[23] Maseko, F.C., Chirwa, M.L. and Muula, A.S. (2014) Client Satisfaction with Cervical Cancer Screening in Malawi. BMC Health Service Research, 14, Article No. 420. https://doi.org/10.1186/1472-6963-14-420

[24] Belete, N., Tsige, Y. and Mellie, H. (2015) Willingness and Acceptibility of Cervical Cancer Screening among Women Living with HIV/AIDS in Addis Abbaba, Ethiopia: A Cross Sectional Study. Gynecologic Oncology Research and Prictice, 2, Article No. 6. https://doi.org/10.1186/s40661-015-0012-3

[25] Bekolo, C.E. and O’bryan, G.T.K.B. (2016) Integrating Cervical Cancer Screening with HIV-Care in Cameroon: Comparative Risk Analysis of Cervical Disease in HIV-Infected Women Receiving Antiretroviral Therapy to Women in the General Population. PLoS ONE, 11, e0149152. https://doi.org/10.1371/journal.pone.0149152

[26] Muthoni, M.A., Ochieng, O.G., Mburugu, K.R, Samson, N., Taratisio, N. and Rose, G. (2016) Socio-Demographic Characteristics Influencing Uptake of Screening for Cervical Cancer in Women Aged 18-49 Years in Imenti North Sub-County, Meru County, Kenya. Science Journal of Public Health, 4, 94-99.

[27] Jones, K.O., Denham, B.E. and Springston, J.K. (2006) Effects of Mass and Interpersonal Communication on Breast Cancer Screening: Advancing Agenda-Setting 
Theory in Health Contexts. Journal of Applied Communication Research, 34, 94-113. https://doi.org/10.1080/00909880500420242

[28] Duffy, F.D., Gordon, G.H, Whelan, G., Cole-kelly, K., Frankel, R. and Skills, I. (2004) Assessing Competence in Communication and Interpersonal Skills: The Kalamazoo II Report. Academic Medicine, 79, 495-507.

https://doi.org/10.1097/00001888-200406000-00002

[29] Brewer, N.T. and Fazekas, K.I. (2007) Predictors of HPV Vaccine Acceptability: A Theory-Informed, Systematic Review. Preventive Medicine, 45, 107-114. https://doi.org/10.1016/j.ypmed.2007.05.013

[30] Gottlieb, S.L., Brewer, N.T. and Sternberg, M.R. (2009) Human Papillomavirus Vaccine Initiation in an Area with Elevated Rates of Cervical Cancer. Journal of Adolescent Health, 45, 430-437. https://doi.org/10.1016/j.jadohealth.2009.03.029 